\title{
Prognostic value of neutrophil-to-lymphocyte ratio, lactate dehydrogenase, D-dimer and CT score in patients with COVID-19
}

\section{Yu-Qing Cai}

Zhongshan Hospital Xiamen University

Hui-Qing Zeng ( $\boldsymbol{\sigma}$ zhq20071212@xmu.edu.cn )

Zhongshan Hospital Xiamen University

Xiao-Bin Zhang

Zhongshan Hospital Xiamen University

Xiao-Jie Wei

Fujian University of Traditional Chinese Medicine

Lan Hu

Tongji Hospital of Tongji Medical College of Huazhong University of Science and Technology

Zhen-Yu Zhang

Zhongshan Hospital Xiamen University

Quan Ming

Yichang Third people's Hospital

Qiu-Ping Peng

Yichang Third people's Hospital

Li-Da Chen

Zhangzhou Municipal Hospital of Fujian Province and Zhangzhou Affiliated Hospital of Fujian Medical University

\section{Research}

Keywords: COVID-19, neutrophil-to-Lymphocyte Ratio, lactate dehydrogenase, D-dimer, CT score

Posted Date: June 30th, 2020

DOI: https://doi.org/10.21203/rs.3.rs-38585/v1

License: (a) (1) This work is licensed under a Creative Commons Attribution 4.0 International License. Read Full License 


\section{Abstract \\ Background}

To explore the significance of neutrophil-to-lymphocyte ratio (NLR), lactate dehydrogenase (LDH), D-dimer and CT score in evaluating the severity and prognosis of coronavirus disease - 2019 (COVID-19).

\section{Methods}

Patients with laboratory confirmed COVID-19 were retrospectively enrolled. The baseline data, laboratory findings, chest computed tomography (CT) results evaluating by CT score on admission, and clinical outcomes were collected and compared. The logistic regression was used to assess the independent relationship between the baseline level of four indicators (NLR, LDH, D-dimer, CT score) and the severity of COVID-19.

\section{Results}

Among 432 patients, 125 (28.94\%) cases were divided into severe group, the remaining $(n=307,71.06 \%)$ were in non-severe group. In multivariate logistic regression, high level of NLR, LDH were independent predictor of the severe group in COVID-19 $(\mathrm{OR}=2.163 ; 95 \% \mathrm{Cl}=1.162-4.026 ; p=0.015$ for $\mathrm{NLR}>3.82 ; \mathrm{OR}=2.298 ; 95 \% \mathrm{Cl}=1.327-$ 3.979; $p=0.003$ for LDH > 246U/L). Combining NLR >3.82 and LDH > 246U/L increased the sensitivity of diagnosis in severe patients (NLR > 3.82 [50.40\%] vs. Combined diagnosis [72.80\%]; $p=0.0007 ; \mathrm{LDH}>246$ [59.2\%] vs. Combined diagnosis [72.80\%]; $p<0.0001)$.

\section{Conclusions}

High levels of NLR and LDH in serum have potential value in the early identification of severe patients with COVID-19. The combination of LDH and NLR can improve the sensitivity of diagnosis.

\section{Introduction}

Since December 2019, coronavirus disease-2019 (COVID-19) caused by a novel coronavirus (SARS-CoV-2) spread rapidly all over the world and has caused a major public health issue (1). By the morning of May 11, Beijing time, the number of patients has exceeded 4.1 million (2). It is obviously a huge challenge to the global healthcare system(3). And the mortality of COVID-19 patients is related to the health-care burden(4).Therefore, the reasonable distribution of medical resources is particularly important. Early identification of critical patients is critical to the rational allocation of resources and the improvement of patients' prognosis.

Meanwhile, according to reports, compared with non-severe patients, the hematological changes of severe patients are more prominent(5). Neutrophil-to-Lymphocyte Ratio (NLR), lactate dehydrogenase (LDH), and Ddimer are close with the poor prognosis of COVID-19(6,7). Without other clinical parameters, the CT evaluation is an independent prognostic factor in patients with COVID-19 (8). However, there is little data about the comparison of the above four indicators. Therefore, in this study, we aimed to compare the prediction efficiency 
of four indicators and evaluate the significance of optimum cutoff. Subsequently, combined diagnosis analysis was performed to evaluate whether it can improve the diagnosis efficiency.

\section{Materials And Methods}

\section{Study Design and Participants}

From January 20, 2020, to March 30, 2020, a total of 432 patients confirmed COVID-19 by the laboratory in designated treatment hospitals (Optic Valley division of Tongji Hospital, Tongji Medical College, Huazhong University of Science and Technology, Wuhan and Yichang Third People's Hospital, Hubei Province) were enrolled The patients were divided into 2 groups based on the seventh edition of the New Coronavirus Pneumonia Diagnosis and Treatment Program published by the Chinese National Health Commission(9): the mild and moderate types were classified as non-severe group and the severe and critical were included into severe group. The disease is classified as severe if one of the following items is met: 1) shortness of breath, respiratory rate $\geq 30$ beats per $\mathrm{min} ; 2$ ) the oxygen saturation $\leq 93 \%$ in a resting state; 3 ) arterial partial pressure of oxygen $\left(\mathrm{PaO}_{2}\right) /$ concentration of oxygen $\left(\mathrm{FiO}_{2}\right) \leq 300 \mathrm{mmHg}(1 \mathrm{mmHg}=0.133 \mathrm{kPa})$; 4) pulmonary images show that the lesions progressed more than $50 \%$ within $24-48 \mathrm{~h}$. The critical should meet one of the following conditions: 1) respiratory failure and need mechanical ventilation; 2) shock; and 3) other organ failures need ICU monitoring and treatment.

\section{Date Collection}

The data of patients' demographic characteristics, comorbidities, laboratory findings, chest computed tomography (CT) results, and clinical outcomes were extracted from electronic medical records. According to the extent of involvement of each lobe, each lobe was scored as 0 (0\%), 1 (1-25\%), 2 (26-50\%), 3 (51-75\%), or 4 (76$100 \%$ ). The total severity score (TSS) is the cumulative score of five lobes (score range 0-20) (10) (11). In order to ensure the accuracy of the data, all data were checked by two physicians, respectively.

\section{Statistical Analysis}

According to the different data distribution, continuous variables were described as mean \pm standard or median (Inter-quartile range, IQR), and groups were compared by student's t-test or Mann-Whitney U test based on the data distribution. Categorical variables were presented as n (\%) and analyzed by Pearson's chi-square. Receiver operator characteristic (ROC) was used to evaluate the efficacy of NLR, LDH, D-dimer and CT score and get the optimum cutoff. Logistic regression was used to access the predictive value for disease risk. The statistical software needed is SPSS version 21 and Medcalc (version 19.1). A value of $p<0.05$ was considered statistically significant.

\section{Results}

\section{Baseline, laboratory and imaging characteristics}

In this retrospective study, a total of 432 patients with COVID-19 were enrolled, including 202(46.5\%) women and $230(53 \%)$ men, the average age was 52.88 years. Fever $(308,71.3 \%)$, cough $(270,62.5 \%)$, expectoration (130, 
$30.1 \%)$ and fatigue $(128,29.6 \%)$ were the common symptoms. Hypertension $(92,21.3 \%)$ was the most common comorbidity.

The patients were divided into two groups: severe group (125/432, 28.94\%) and non-severe (307/432, 71.06\%) group based on the severity of the disease. Comparing with the non-severe group, in terms of the baseline characteristics, the average age of severe group was older (59.60 \pm 16.65 years vs $50.14 \pm 16.26$ years, $p<0.0001)$. Meanwhile, it is noticed that severe group has higher incidence of comorbidities, such as hypertension $(p<0.0001)$, diabetes $(p<0.0001)$, and COPD ( $p=0.009$. As for the clinical laboratory findings, lower level of lymphocyte $(p<0.0001)$ and higher level of white blood cell $(p=0.023)$, neutrophil $(p<0.0001)$, C-reaction protein $(p<0.0001)$, LDH $(p<0.0001)$, D-dimer $(p<0.0001)$ and NLR $(p<0.0001)$ were detected in severe group as compared with non-severe group. Regarding CT results, 96.0\% (120/125) patients had bilateral lung involvement, 32\% (40/125) consolidation, and 3.2\% (4/125) pleural effusion among patients in severe group. There was significant difference in CT score (6.0 [4-9] for severe group vs 6[4-7] for non-severe group, $p<0.0001)$ between two groups (Table 1).

\section{Predictive value of NLR, LDH, D-dimer and CT score}

As table 1 showed, NLR, LDH, D-dimer, and CT score were statistically significantly higher in the severe group. On the basis of receiver operating characteristic (ROC), the area under curve (AUC) was 0.716 for NLR, 0.740 for $\mathrm{LDH}, 0.650$ for D-dimer, and 0.612 for CT score, indicating certain diagnostic value for the severity of disease (Figure 1 and Table 2). In addition, the optimum cutoff from ROC was 3.82, $246 \mathrm{U} / \mathrm{L}, 0.83 \mu \mathrm{g} / \mathrm{ml}$, and 7 for NLR, LDH, D-dimer, and CT score, respectively (Table 2).

We assumed that when the level of NLR, LDH, D-dimer, and CT score on admission exceeded the optimum cutoff, the patients were prone to develop severe or critical types. According to optimum cutoff, the patients were divided into different subgroups.

As table 3 showed, about 25.9\% (112/432), 31.9\% (138/432), 22.2\% (96/432) and 25\% (108/432) patient, respectively, had high level of NLR, LDH, D-dimer and CT score on admission. After grouping, the distribution of baseline NLR [63/125 (50.4\%) vs 49/307(16\%); $p<0.0001]$, LDH [74/125(59.2\%) vs. 64/307(20.8\%); $p<0.0001]$; Ddimer[47/125 (37.6\%) vs 49/307(16\%); $p<0.0001]$ and CT score [46/125 (36.8\%) vs 62/307 (20.2\%); $p<0.0001]$ over optimum cutoff in two groups were significantly significant (Table 3 ).

Univariate analysis indicated that the high level of NLR, LDH, D-dimer and CT score positively correlated with the severity of disease $(\mathrm{OR}=5.350 ; 95 \% \mathrm{Cl}=3.361-8.518 ; p<0.0001$ for $\mathrm{NLR} ; \mathrm{OR}=5.509 ; 95 \% \mathrm{Cl}=3.511-8.646 ; p<0.0001$ for LDH; OR=3.173; 95\%Cl=1.976-5.094; $p<0.0001$ for D-dimer; OR=2.301; 95\%Cl=1.455-3.638; $p<0.0001$ for CT score). However, after adjusting other statistically significant index, the predictive value of NLR $>3.82$, $\mathrm{LDH}>246 \mathrm{U} / \mathrm{L}$ were persisted $(\mathrm{OR}=2.163 ; 95 \% \mathrm{Cl}=1.162-4.026 ; p=0.015$ for $\mathrm{NLR} ; \mathrm{OR}=2.298 ; 95 \% \mathrm{Cl}=1.327-3.979$; $p=0.003$ for $\mathrm{LDH}$ ). While the relationship between $\mathrm{D}$-dimer $>0.83 \mu \mathrm{g} / \mathrm{ml}, \mathrm{CT}$ score $>7$ and the severity of disease was weakened $(\mathrm{OR}=1.209 ; 95 \% \mathrm{Cl}=0.626-2.334 ; p=0.571$ for $\mathrm{D}$-dimer; $\mathrm{OR}=1.519 ; 95 \% \mathrm{Cl}=0.71-3.247 ; p=0.281$ for CT score). In addition, fatigue (OR=1.978;95\% Cl=1.127-3.473; $p=0.018)$, chest tightness $(\mathrm{OR}=2.265 ; 95 \% \mathrm{Cl}=$ $1.011-5.074 ; p=0.047)$, hypertension $(\mathrm{OR}=2.534,95 \% \mathrm{Cl}=1.259-5.099 ; p=0.009), \mathrm{CRP}(\mathrm{OR}=1.013 ; 95 \% \mathrm{Cl}=1.003-$ $1.023 ; p=0.011)$, bilateral lung involved $(\mathrm{OR}=3.890 ; 95 \% \mathrm{Cl}=1.356-11.154 ; p=0.011)$ were still positively correlated with the severity of disease (Table 4). 


\section{Evaluation of multi-parameter model}

According to the logistic regression, NLR $>3.82$ and $L D H>246 \mathrm{U} / \mathrm{L}$ were statistically significant risk factors (Table 4). And as table 2 showed, the sensitivity of NLR $>3.82$ and $L D H>246 \mathrm{U} / \mathrm{L}$ in predicting the severity of COVID-19 were $50.40 \%$ and $59.20 \%$, respectively. Then, further evaluation was made to judge whether the combined diagnosis model of two indexes can improve the sensitivity of prediction.

The table 5 indicated that the combined diagnosis of $\mathrm{NLR}>3.82$ and $L D H>246 \mathrm{U} / \mathrm{L}$ could increase the sensitivity in predicting the severity of disease [NLR $>3.82(50.40 \%) v s$ combined diagnosis model $(72.80 \%) ; p=0.0007$; $\mathrm{LDH}>246(59.2 \%)$ vs combined diagnosis model $(72.80 \%) ; p<0.0001]$.

\section{Discussion}

A total of 432 patients with COVID-19 were included in this retrospective study. In the univariate analysis, we found that the high level of NLR, LDH, D-dimer, and CT score have significant correlation with the severity of COVID-19. While after adjusting other statistically significant indexes, the predictive value of NLR > 3.82, LDH > $246 \mathrm{U} / \mathrm{L}$ were persisted. This indicated that when NLR exceeded the cutoff point, the risk of serious disease increased by 2.163 times. And the risk of LDH over optimum cutoff increased by 2.298 times. While the value of D-dimer $>0.83 \mu \mathrm{g} / \mathrm{ml}$ and CT score $>7$ in predicting the severity of disease was weak and could not be recommended as independent predictors. In addition, the risk of severity was also closely related to fatigue, chest tightness, hypertension and CRP. Meanwhile, combining NLR $>3.82$ and LDH $>246 \mathrm{U} / \mathrm{L}$ can improve the sensitivity of disease risk prediction.

Immune dysfunction plays an important role in the severity of COVID-19 (12). Recent studies have elucidated that neutropenia and lower lymphopenia could be found in the severe group of COVID-19(13,14). NLR took lymphocyte and neutrophil into account at the same time. Several studies have shown the predictive value of NLR in distinguishing COVID-19 patients with severe and critical types. In a study of the dynamic changes of lymphocyte subsets and cytokine profiles in patients with COVID-19, NLR can be used as a prognostic factor for early identification of severe cases(15). A cohort of patients with COVID-19 also proved that, after adjustment of confounding factors, each unit increase in NLR, the risk of in-hospital mortality increases by $8 \%(16)$. Another study conducted by Yang $X$ et. al, (6) in 93 patients with COVID-19 demonstrated that NLR can be used as independent indicators for poor clinical outcome and the largest AUC for NLR were 0.841 , with specificity (63.6\%) and sensitivity (88\%). However, limited by sample diversity, the outcome needs further evaluation. In the present study, the predictive value of NLR is consistent with abovementioned studies. Meanwhile, the sample size and diversity were enriched by collecting data from two clinical centers, which will strengthen the reliability of conclusions. The optimum cutoff for NLR was 3.82 and AUC was 0.716 . And the sensitivity and specificity of NLR $>3.82$ were $50.40 \%$ and $84.04 \%$, respectively. Meanwhile, in multivariate logistic regression, NLR $>3.82$ can be used as an independent predictor for disease risk $(\mathrm{OR}=2.163 ; 95 \% \mathrm{Cl}=1.162-4.026 ; p=0.015)$.

The elevation of LDH was one of the most common laboratory abnormalities in patients with COVID-19. Acute lung injury was highly related to $\mathrm{LDH}(17)$. A systematic literature review and meta-analysis had shown that LDH $>245 \mathrm{U} / \mathrm{L}$ can predict the progress of COVID-19(7). In a study of the risk factors for death in cancer patients with COVID-19, the elevated LDH was closely related to the increase of mortality (18). Furthermore, in another retrospective analysis of 120 patients with COVID-19, comparing with mild patients, the severe patients have 
higher LDH levels (mean 200.8 U/L for mild vs mean 342.8 U/L for severe)(19). The predictive value of LDH is further confirmed by our study. Meanwhile, ROC analysis showed that the AUC for LDH was 0.74 and the optimum cutoff was $246 \mathrm{U} / \mathrm{L}$. The sensitivity was $59.2 \%$ and the specificity was $79.15 \%$. And the logistic regression indicated that the risk of serious disease increased by 2.298 times when LDH over optimum cutoff $(\mathrm{OR}=2.298 ; 95 \% \mathrm{Cl}=1.327-3.979 ; p=0.003)$. In addition, the sensitivity of disease risk prediction can be improved by combining LDH > 246U/L with NLR > 3.82. (NLR > 3.82 [50.40\%] vs. combined diagnosis model [72.80\%]; $p=0.0007 ; \mathrm{LDH}>246$ [59.2\%] vs. combined diagnosis model [72.80\%]; $p<0.0001)$. However, the specificity were decreased (NLR > 3.82 [84.04\%] vs. combined diagnosis model [69.71\%]; $p=0.0007 ;$ LDH > 246[79.15\%] vs. combined diagnosis model [69.71\%]; $p<0.0001)$.

Moreover, the sensitivity, specificity, and AUC for NLR and LDH are not relatively high enough. Due to the different admission time of patients with COVID-19 and the acute aggravation of some patients in a period of time after admission, the value of admission indicators may be underestimated. However, compared with other articles(6, $15,20)$, the sample size and diversity of patients with COVID-19 increase the reliability of the results in this study. Meanwhile, more importantly, the optimum cutoff can indicate the risk of acute aggravation of patients with COVID-19 in the present study. Furthermore, it provides more evidence for the establishment of multi-parameter diagnosis model.

Coagulation disorders are more common in severe patients than in light patients $(21,22)$. A study conducted by Zhang $L$ et al.(23) had proved that the level of $D$-dimer $\geq 2.0 \mu \mathrm{g} / \mathrm{mL}$ (fourfold increase) could effectively predict the mortality of patients with COVID-19. While after balancing the confounding factors, the logistic regression showed that D-dimer $>0.83 \mu \mathrm{g} / \mathrm{ml}$ could not be used as an independent predictor of disease risk in this study $(\mathrm{OR}=1.209 ; 95 \% \mathrm{Cl}=0.626-2.334 ; p=0.571)$. In a dynamic study of hematological parameters in patients with COVID-19, the D-dimer of the severe group was higher than the non-severe group on days 1,7 and $14(p<0.05)$ (24). This suggests that due to different admission times, the ability of D-dimer to predict disease risk may be weakened. In another response to the prognostic value of D-dimer in patients with COVID-19, the predictive value of D-dimer might be affected by other factors, such as hormonotherapy, antibiotic therapy et al. Due to the baseline level of D-dimer varies greatly in patients, the value of dynamic monitoring of D-dimer may be higher in patients with COVID-19(25). Further researches are still required to evaluate the significance of D-dimer in evaluating the severity of COVID-19.

COVID-19 patients have lung involvement with imaging changes $(10,26)$. In different stages of the disease, the CT manifestations are different, which are important to the diagnosis and staging of patients (27). With the same semi-quantitative scoring system, a multi-center paired cohort study conducted by J. Liu et al. (28) showed that CT changes are obvious in acute exacerbation of COVID-19, accompanied by an increase of CT score. This indicated that elevated CT score may predict the poor outcome. Another retrospective single-center study indicated that the CT score has a high diagnostic value in patients with severe COVID-19. ROC analysis showed that AUC for CT score was 0.918 . The optimum cutoff of CT score was 7.5 . The sensitivity was $82.6 \%$ and the specificity was $100 \%(11)$. However, the study only analyzes imaging, without combined with clinical data. And significant differences in the number of patients between severe-critical patients and non-severe groups also affect the accuracy of the results. While in present study, after combining the clinical data, the CT score can't be used as an independent predictor of disease risk $(\mathrm{OR}=1.519 ; 95 \% \mathrm{Cl}=0.71-3.247 ; p=0.281)$. A study by Zhang $B$ et. al(29), demonstrated that the severity of lung abnormalities evaluated by CT score might be associated with laboratory parameters. Therefore, due to correlation between CT score and laboratory parameters, the ability to 
independently predict disease risk of CT score may be attenuated. Additional investigations are warranted to assess whether CT score can be an independent predictor of disease risk.

There are some limitations in this study. First, owing to different severity of patients and different medical resources, the time from onset to admission might not be representative, which might affect the level of four parameters on admission. Meanwhile, the representativeness of CT score and D-dimer may also be affected by different admission times. Second, other clinical data and test results are not included in the analysis, which may cause bias, weakening the reliability of the results. Third, to a certain degree, the CT score as a semi-quantitative evaluation method was subjective.

\section{Conclusion}

As independent factors, the levels of NLR, LDH in serum have a significant correlation with the severity of COVID19. We suggested that NLR and LDH could be recommended as predictors for evaluating the severity of COVID19.

\section{Declarations}

Ethics approval and consent to participate: The study was approved by the Ethics Committee of Zhongshan Hospital, Xiamen University and Second affiliated Hospital of Fujian Medical University.

Consent for publication: No applicable.

Availability of data and material: All data generated or analyzed during this study are included in this published article.

Competing interests: The authors declare that they have no conflict of interest.

Funding: This work was supported by Grant 2018-2-65 for Youth Research Fund from Fujian Provincial Health Bureau, and Grant 2018J01393 for Fund from Natural Science Foundation of Fujian Province, China.

Authors' contributions: Conception and design: Y-Q Cai, X-B Zhang, and H-Q Zeng. Collection and assembly of data: Y-Q Cai, X-B Zhang, X-J Wei, Z-Y Zhang, L-D Chen, L Hu, Q Ming, Q-P Peng .Data analysis and interpretation: $\mathrm{Y}-\mathrm{Q}$ Cai, X-B Zhang, and H-Q Zeng. Manuscript writing: All authors. Final approval of manuscript: All authors.

Acknowledgments: The authors would like to thank Jihong Liu, dean of Tongji Hospital Affiliated to Tongji Medical College of Huazhong University of science and technology, for the support in data collection.

\section{References}

1. Lai CC, Shih TP, Ko WC, Tang HJ, Hsueh PR. Severe acute respiratory syndrome coronavirus 2 (SARS-CoV-2) and coronavirus disease-2019 (COVID-19): The epidemic and the challenges. Int J Antimicrob Agents. 2020;55(3):105924.

2. The diagnosis of the novel. coronavirus pneumonia exceeds 4.1 million British and French gradually "unsealed": The China News Service; 2020-05-11 [Available from: https://baijiahao.baidu.com/s? id $=1666376262699977033 \&$ wfr $=$ spider \&for $=p c$.

Page $7 / 13$ 
3. Verelst F, Kuylen E, Beutels P. Indications for healthcare surge capacity in European countries facing an exponential increase in coronavirus disease (COVID-19) cases, March 2020. Euro Surveill. 2020;25(13).

4. Ji Y, Ma Z, Peppelenbosch MP, Pan Q. Potential association between COVID-19 mortality and health-care resource availability. The Lancet Global Health. 2020;8(4).

5. Velavan TP, Meyer CG. Mild versus severe COVID-19: laboratory markers. Int J Infect Dis. 2020.

6. Yang $X, Y u$ Y, Xu J, Shu H, Xia J, Liu H, et al. Clinical course and outcomes of critically ill patients with SARSCoV-2 pneumonia in Wuhan, China: a single-centered, retrospective, observational study. Lancet Respir Med. 2020.

7. Zheng Z, Peng F, Xu B, Zhao J, Liu H, Peng J, et al. Risk factors of critical \& mortal COVID-19 cases: A systematic literature review and meta-analysis. Journal of Infection. 2020.

8. Colombi D, Bodini FC, Petrini M, Maffi G, Morelli N, Milanese G, et al. Well-aerated Lung on Admitting Chest CT to Predict Adverse Outcome in COVID-19 Pneumonia. Radiology. 2020:201433.

9. Diagnosis and Treatment of Pneumonia of New Coronavirus Infection (Trial Version 7). National Health and Health Commission of the people's Republic of China; 2020-03-03 [Available from:

http://www.nhc.gov.cn/yzygj/s7653p/202003/46c9294a7dfe4cef80dc7f5912eb1989.shtml.

10. Chung M, Bernheim A, Mei X, Zhang N, Huang M, Zeng X, et al. CT Imaging Features of 2019 Novel Coronavirus (2019-nCoV). Radiology. 2020;295(1):202-7.

11. Li K, Fang Y, Li W, Pan C, Qin P, Zhong Y, et al. CT image visual quantitative evaluation and clinical classification of coronavirus disease (COVID-19). Eur Radiol. 2020.

12. Giamarellos-Bourboulis EJ, Netea MG, Rovina N, Akinosoglou K, Antoniadou A, Antonakos N, et al. Complex Immune Dysregulation in COVID-19 Patients with Severe Respiratory Failure. Cell Host \& Microbe. 2020.

13. Qin C, Zhou L, Hu Z, Zhang S, Yang S, Tao Y, et al. Dysregulation of immune response in patients with COVID-19 in Wuhan, China. Clin Infect Dis. 2020.

14. Jiang M, Guo Y, Luo Q, Huang Z, Zhao R, Liu S, et al. T cell subset counts in peripheral blood can be used as discriminatory biomarkers for diagnosis and severity prediction of COVID-19. J Infect Dis. 2020.

15. Liu J, Li S, Liu J, Liang B, Wang X, Wang H, et al. Longitudinal characteristics of lymphocyte responses and cytokine profiles in the peripheral blood of SARS-CoV-2 infected patients. EBioMedicine. 2020;55.

16. Liu Y, Du X, Chen J, Jin Y, Peng L, Wang HHX, et al. Neutrophil-to-lymphocyte ratio as an independent risk factor for mortality in hospitalized patients with COVID-19. Journal of Infection. 2020.

17. Liu Y, Yang Y, Zhang C, Huang F, Wang F, Yuan J, et al. Clinical and biochemical indexes from 2019-nCoV infected patients linked to viral loads and lung injury. Sci China Life Sci. 2020;63(3):364-74.

18. Mehta V, Goel S, Kabarriti R, Cole D, Goldfinger M, Acuna-Villaorduna A, et al. Case Fatality Rate of Cancer Patients with COVID-19 in a New York Hospital System. Cancer Discov. 2020.

19. Zhang R, Ouyang H, Fu L, Wang S, Han J, Huang K, et al. CT features of SARS-CoV-2 pneumonia according to clinical presentation: a retrospective analysis of 120 consecutive patients from Wuhan city. Eur Radiol. 2020.

20. Xia X, Wen M, Zhan S, He J, Chen W. [An increased neutrophil/lymphocyte ratio is an early warning signal of severe COVID-19]. Nan Fang Yi Ke Da Xue Xue Bao. 2020;40(3):333-6.

21. Tang N, Li D, Wang X, Sun Z. Abnormal coagulation parameters are associated with poor prognosis in patients with novel coronavirus pneumonia. J Thromb Haemost. 2020;18(4):844-7. 
22. Terpos E, Ntanasis-Stathopoulos I, Elalamy I, Kastritis E, Sergentanis TN, Politou M, et al. Hematological findings and complications of COVID-19. Am J Hematol. 2020.

23. Zhang L, Yan X, Fan Q, Liu H, Liu X, Liu Z, et al. D-dimer levels on admission to predict in-hospital mortality in patients with Covid-19. J Thromb Haemost. 2020.

24. Fu J, Kong J, Wang W, Wu M, Yao L, Wang Z, et al. The clinical implication of dynamic neutrophil to lymphocyte ratio and D-dimer in COVID-19: A retrospective study in Suzhou China. Thromb Res. 2020;192:3-8.

25. Zhang L. Response to "uncertainties on the prognostic value of D-dimers in COVID-19 patients". J Thromb Haemost. 2020.

26. Chen N, Zhou M, Dong X, Qu J, Gong F, Han Y, et al. Epidemiological and clinical characteristics of 99 cases of 2019 novel coronavirus pneumonia in Wuhan, China: a descriptive study. The Lancet. 2020;395(10223):507-13.

27. Li M, Lei P, Zeng B, Li Z, Yu P, Fan B, et al. Coronavirus Disease (COVID-19): Spectrum of CT Findings and Temporal Progression of the Disease. Academic Radiology. 2020;27(5):603-8.

28. Liu J, Chen T, Yang H, Cai Y, Yu Q, Chen J, et al. Clinical and radiological changes of hospitalised patients with COVID-19 pneumonia from disease onset to acute exacerbation: a multicentre paired cohort study. Eur Radiol. 2020.

29. Zhang B, Zhang J, Chen H, Chen L, Chen Q, Li M, et al. Novel coronavirus disease 2019 (COVID-19): relationship between chest CT scores and laboratory parameters. Eur J Nucl Med Mol Imaging. 2020.

\section{Tables}

Table 1: Baseline, laboratory and imaging characteristics 


\begin{tabular}{lllll}
\hline Variable & $\begin{array}{l}\text { Total } \\
(\mathrm{n}=432)\end{array}$ & $\begin{array}{l}\text { Severe group } \\
(\mathrm{n}=125)\end{array}$ & $\begin{array}{l}\text { Non-severe } \\
\text { Group }(\mathrm{n}=307)\end{array}$ & $p$ value \\
\hline Age(years) & $52.88 \pm 16.91$ & $59.60 \pm 16.65$ & $50.14 \pm 16.26$ & $<0.0001$ \\
\hline Gender & & & & 0.072 \\
\hline Female-n (\%) & $202(46.5)$ & $50(40)$ & $152(49.5)$ & \\
\hline Male-n (\%) & $230(53)$ & $75(60)$ & $155(50.5)$ & \\
\hline Clinical symptom-n (\%) & & & $214(69.7)$ & 0.252 \\
\hline Fever & $308(71.3)$ & $94(75.2)$ & $82(26.7)$ & 0.037 \\
\hline Fatigue & $128(29.6)$ & $46(36.8)$ & $15(4.9)$ & $<0.0001$ \\
\hline Dyspnea & $35(8.1)$ & $20(16)$ & $22(7.2)$ & 0.394 \\
\hline Pharyngalgia & $34(7.9)$ & $12(9.6)$ & $186(60.6)$ & 0.198 \\
\hline Cough & $270(62.5)$ & $84(67.2)$ & $25(8.1)$ & 0.004 \\
\hline Chest tightness & $47(10.9)$ & $22(17.6)$ & $14(4.6)$ & 0.914 \\
\hline Diarrhea & $20(4.6)$ & $6(4.8)$ & $27(8.8)$ & 0.05 \\
\hline Myalgia & $46(10.6)$ & $19(15.2)$ & $95(30.9)$ & 0.545 \\
\hline Expectoration & $130(30.1)$ & $35(28)$ & $13(4.2)$ & 0.795 \\
\hline Headache & $19(4.4)$ & $6(4.8)$ & $34(11.1)$ & 0.236 \\
\hline Poor appetite & $53(12.3)$ & $19(15.2)$ & &
\end{tabular}

Comorbidities-n[\%ם

\begin{tabular}{|c|c|c|c|c|}
\hline Hypertension & $92(21.3)$ & $53(42.4)$ & $39(12.7)$ & $<0.0001$ \\
\hline Diabetes & $56(13)$ & $31(24.8)$ & $25(8.1)$ & $<0.0001$ \\
\hline COPD & $25(5.8)$ & $13(10.4)$ & $12(3.9)$ & 0.009 \\
\hline Renal insufficiency & $9(2.1)$ & $8(6.4)$ & $1(0.3)$ & $<0.0001$ \\
\hline Cardiac insufficiency & $8(1.9)$ & $7(5.6)$ & $1(0.3)$ & 0.01 \\
\hline Hepatic insufficiency & $30(6.9)$ & $16(12.8)$ & $14(4.6)$ & 0.002 \\
\hline Anemia & $13(3.0)$ & $7(5.6)$ & $6(2.0)$ & 0.089 \\
\hline \multicolumn{5}{|l|}{ Clinical laboratory } \\
\hline White blood cell-10^9$/ \mathrm{L}$ & $5.25 \pm 2.52$ & $5.76 \pm 3.19$ & $5.04 \pm 2.16$ & 0.023 \\
\hline Lymphocyte- $10^{\wedge} 9 / \mathrm{L}$ & $1.28 \pm 0.62$ & $1.04 \pm 0.70$ & $1.37 \pm 0.55$ & $<0.0001$ \\
\hline Neutrophil-10^9/L (IQR) & $2.98(2.11-4.18)$ & $3.41(2.32-5.50)$ & $2.82(2.08-3.77)$ & $<0.0001$ \\
\hline CRP-mg/L(IQR) & $22.32(9.15-37.7)$ & $45.2(14.85-55.8)$ & $22.32(7.7-22.32)$ & $<0.0001$ \\
\hline Platelet-10^9$/ \mathrm{L}$ & $173.02 \pm 80.88$ & $163.03 \pm 83.74$ & $177.08 \pm 79.47$ & 0.102 \\
\hline D-dimer- $\mu \mathrm{g} / \mathrm{ml}(\mathrm{IQR})$ & $0.55(0.44-0.82)$ & $0.62(0.50-1.42)$ & $0.52(0.42-0.68)$ & $<0.0001$ \\
\hline & & 265(207.5-356) & 196(162-235) & $<0.0001$ \\
\hline NLR (IQR) & $2.33(1.51-3.94)$ & $3.84(2.06-7.13)$ & $2.03(1.41-3,25)$ & $<0.0001$ \\
\hline \multicolumn{5}{|l|}{ CT manifestations } \\
\hline $\begin{array}{l}\text { CT score (IQR) } \\
\text { Bilateral lung involved-n (\%) }\end{array}$ & $\frac{6(4-7.75)}{250(021)}$ & $\frac{6(4-9)}{120(06)}$ & $\frac{6(4-7)}{220(779)}$ & $<0.0001$ \\
\hline Bilateral lung involved-n (\%) & $359(83.1)$ & $120(96)$ & $239(77.9)$ & $<0.0001$ \\
\hline Ground glass opacity-n (\%) & $426(98.6)$ & $124(99.2)$ & $302(98.4)$ & 0.830 \\
\hline Consolidation-n (\%) & $96(22.2)$ & $40(32)$ & $56(18.2)$ & 0.002 \\
\hline Pleural effusion-n (\%) & $5(1.2)$ & $4(3.2)$ & $1(0.3)$ & 0.042 \\
\hline Pleural thickening-n (\%) & $5(1.2)$ & $1(0.8)$ & $4(1.3)$ & 1.000 \\
\hline
\end{tabular}


Abbreviation: $\mathrm{COPD}=$ chronic obstructive pulmonary disease, $\mathrm{CRP}=\mathrm{C}$-reactive protein, $\mathrm{NLR}=$ neutrophil - to- lymphocyte ratio, LDH=lactate dehydrogenase.

Table 2 Area under ROC curve and optimum cutoff

\begin{tabular}{|c|c|c|c|c|c|c|c|c|}
\hline \multirow[t]{3}{*}{ Variables } & \multirow{3}{*}{ AUC } & \multicolumn{3}{|c|}{ Assessment of validity } & \multirow{2}{*}{\multicolumn{2}{|c|}{ Predictive value }} & \multirow{2}{*}{\multicolumn{2}{|c|}{ Likelihood ratio }} \\
\hline & & Optimum & Sensitivity & Specificity & & & & \\
\hline & & cutoIf & & & positive & Negative & positive & negative \\
\hline NLR & 0.716 & 3.82 & $50.40 \%$ & $84.04 \%$ & 56.3 & $80.6 \%$ & 3.16 & 0.59 \\
\hline LDH(U/L) & 0.740 & 246 & $59.20 \%$ & $79.15 \%$ & $53.6 \%$ & $82.7 \%$ & 2.84 & 0.52 \\
\hline $\begin{array}{l}\text { D- } \\
\text { dimer }(\mu \mathrm{g} / \mathrm{ml})\end{array}$ & 0.650 & 0.83 & $37.6 \%$ & $84.04 \%$ & $49 \%$ & $76.8 \%$ & 2.36 & 0.74 \\
\hline CT-score & 0.612 & 7 & $36.8 \%$ & $79.8 \%$ & $42.6 \%$ & $75.6 \%$ & 1.82 & 0.79 \\
\hline
\end{tabular}

Abbreviation: $\mathrm{ROC}=$ receiver operator characteristic, NLR=neutrophil-to-lymphocyte ratio, $\mathrm{LDH}=$ lactate dehydrogenase.

\section{Table3 Baseline after grouping}

\begin{tabular}{lllll}
\hline & $\begin{array}{l}\text { Total } \\
\mathrm{n}=432\end{array}$ & $\begin{array}{l}\text { Severe group } \\
\mathrm{n}=125\end{array}$ & $\begin{array}{l}\text { Non -severe group } \\
\mathrm{n}=307\end{array}$ & $p$ value \\
\hline NLR & & & & \\
\hline$>3.82$ & $112(25.9 \%)$ & $63(50.4 \%)$ & $49(16 \%)$ & $p<0.0001$ \\
\hline$\leq 3.82$ & $320(74.1 \%)$ & $62(49.6 \%)$ & $258(84 \%)$ & \\
\hline LDH $(\mathrm{U} / \mathrm{L})$ & & & & \\
\hline$>246$ & $138(31.9 \%)$ & $74(59.2 \%)$ & $64(20.8 \%)$ & \\
\hline$\leq 246$ & $294(68.1 \%)$ & $51(40.8 \%)$ & $243(79.2 \%)$ & \\
\hline D-dimer $(\mu \mathrm{g} / \mathrm{ml})$ & & & & \\
\hline$>0.83$ & $96(22.2 \%)$ & $47(37.6 \%)$ & $49(16 \%)$ & \\
\hline$\leq 0.83$ & $336(77.8 \%)$ & $78(62.4 \%)$ & $258(84 \%)$ & \\
\hline CT Score & & & & \\
\hline$>7$ & $108(25 \%)$ & $46(36.8 \%)$ & $62(20.2 \%)$ & \\
\hline$\leq 7$ & $324(75 \% 0$ & $79(63.2 \%)$ & $245(79.8 \%)$ & \\
\hline
\end{tabular}

Abbreviation: $\mathrm{ROC}=$ receiver operator characteristic, NLR=neutrophil-to-lymphocyte ratio, $\mathrm{LDH}=$ lactate dehydrogenase.

Table 4 The univariate and multivariable logistic regression 


\begin{tabular}{lllll}
\hline Variables & $\begin{array}{l}\text { Unadjusted Odds ratio } \\
(95 \% \mathrm{CI})\end{array}$ & $p$ value & $\begin{array}{l}\text { Adjusted Odds ratio } \\
(95 \% \mathrm{CI})\end{array}$ & $p$ value \\
\hline NLR & $5.350(3.361,8.518)$ & $<0.0001$ & $2.163(1.162,4.026)$ & 0.015 \\
\hline LDH(U/L) & $5.509(3.511,8.646)$ & $<0.0001$ & $2.298(1.327,3.979)$ & 0.003 \\
\hline D-dimer $(\mu \mathrm{g} / \mathrm{ml})$ & $3.173(1.976,5.094)$ & $<0.0001$ & $1.209(0.626,2.334)$ & 0.571 \\
\hline CT score & $2.301(1.455,3.638)$ & $<0.0001$ & $1.519(0.71,3.247)$ & 0.281 \\
\hline Age & $1.036(1.022,1.050)$ & $<0.0001$ & $0.994(0.975,1.014)$ & 0.561 \\
\hline Fatigue & $1.598(1.026,2.488)$ & 0.038 & $1.978(1.127,3.473)$ & 0.018 \\
\hline Dyspnea & $3.708(1.831,7.509)$ & $<0.0001$ & $1.348(0.507,3.585)$ & 0.55 \\
\hline Chest tightness & $2.409(1.302,4.460)$ & 0.005 & $2.265(1.011,5.074)$ & 0.047 \\
\hline Hypertension & $5.058(3.103,8.245)$ & $<0.0001$ & $2.534(1.259,5.099)$ & 0.009 \\
\hline Diabetes & $3.720(2.091,6.619)$ & $<0.0001$ & $1.304(0.597,2.848)$ & 0.506 \\
\hline COPD & $2.853(1.264,6.441)$ & 0.012 & $1.019(0.314,3.303)$ & 0.975 \\
\hline Renal insufficiency & $20.923(2.589,169.118)$ & 0.004 & $4.788(0.449,51.025)$ & 0.195 \\
\hline Cardiac insufficiency & $18.153(2.210,149.133)$ & 0.007 & $2.245(0.135,37.251)$ & 0.573 \\
\hline Hepatic insufficiency & $3.072(1.451,6.505)$ & 0.003 & $2.209(0.842,5.792)$ & 0.107 \\
\hline CRP (mg/L) & $1.025(1.017,1.033)$ & $<0.0001$ & $1.013(1.003,1.023)$ & 0.011 \\
\hline Bilateral lung involved & $6.828(2.683,17.381)$ & $<0.0001$ & $3.890(1.356,11.154)$ & 0.011 \\
& & & & \\
\hline Consolidation & $2.109(1.312,3.390)$ & 0.002 & $1.303(0.6,2.829)$ & 0.504 \\
\hline Pleural effusion & $10.116(1.119,91.421)$ & 0.039 & $5.097(0.409,63.513)$ & 0.206 \\
\hline
\end{tabular}

Abbreviation: NLR = neutrophil - to- lymphocyte ratio, LDH=lactate dehydrogenase, $\mathrm{COPD}=$ chronic obstructive pulmonary disease, $\mathrm{CRP}=\mathrm{C}$-reactive protein.

Table 5 Comparison of univariate and combined diagnosis model

\begin{tabular}{llll}
\hline Variables & $p$ value & Sensitivity & Specificity \\
& & & \\
\hline NLR $>3.82$ & $0.0007^{1}$ & $50.40 \%$ & $84.04 \%$ \\
\hline LDH $>246 U / L$ & $<0.0001^{2}$ & $59.20 \%$ & $79.15 \%$ \\
\hline Combined diagnosis model & & $72.80 \%$ & $69.71 \%$ \\
\hline
\end{tabular}

Abbreviation: NLR = neutrophil - to- lymphocyte ratio, LDH=lactate dehydrogenase, combined diagnosis model $=\mathrm{NLR}>3.82$ and $\mathrm{LDH}>246 \mathrm{U} / \mathrm{L}, 0.0007^{1}=p$ value between $\mathrm{NLR}>3.82$ and combined diagnosis model, $<0.0001^{2}=p$ value between $\mathrm{LDH}>246 \mathrm{U} / \mathrm{L}$ and combined diagnosis model.

\section{Figures}


A

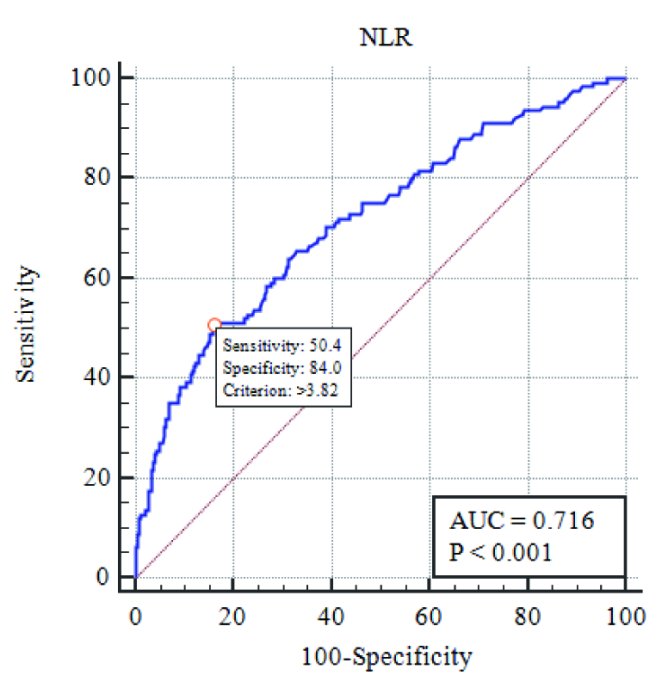

C

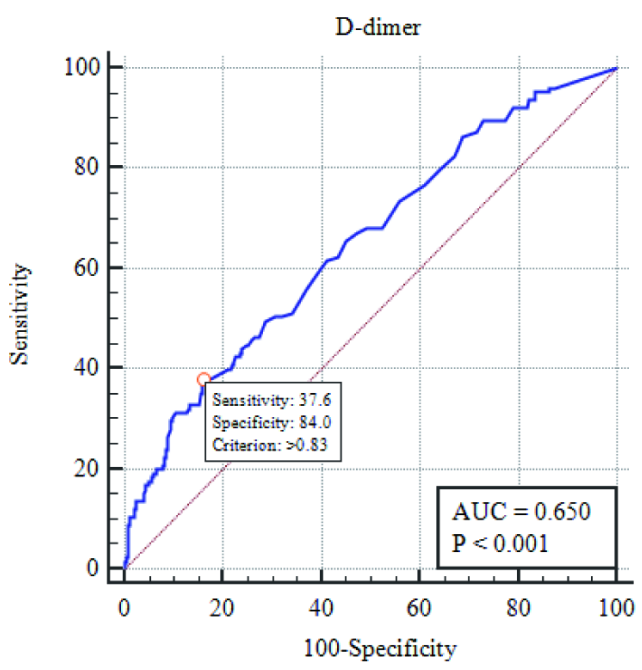

B

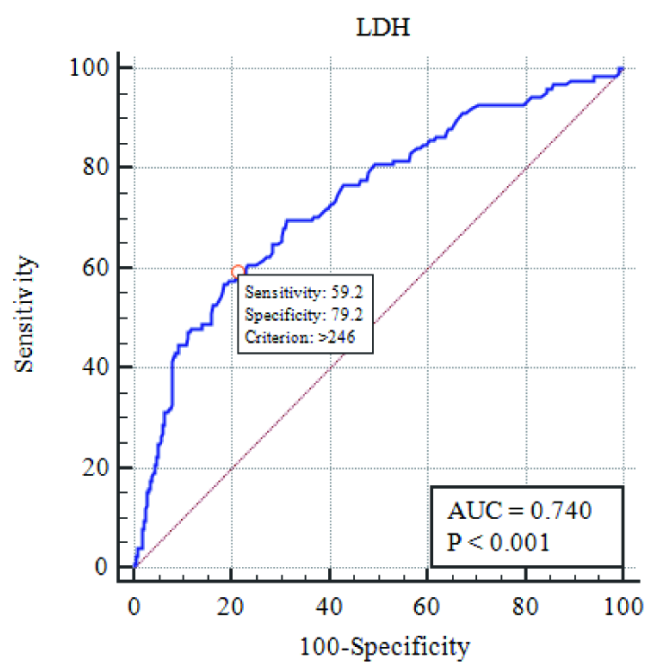

D

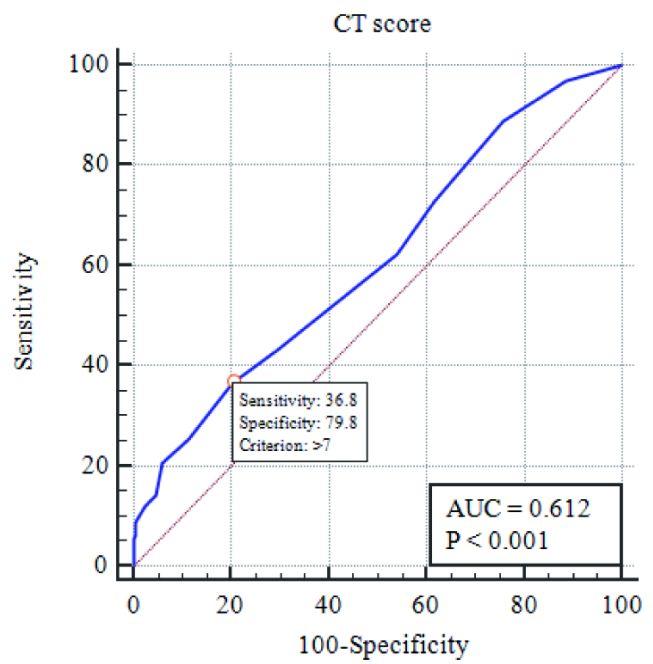

\section{Figure 1}

ROC analysis of NLR, LDH, D-dimer and CT score in disease risk prediction (A. NLR; B.LDH; C. D-dimer; D. CT score). 